\title{
On the construction of the solution of an equation describing an axially moving string.
}

\author{
W.T. van Horssen *, S.V. Ponomareva \\ Department of Applied Mathematical Analysis, \\ Faculty of Information Technology and Systems, \\ Delft University of Technology, \\ Mekelweg 4, 2628 CD Delft, The Netherlands.
}

\begin{abstract}
In this paper an initial-boundary value problem for a linear, nonhomogeneous axially moving string equation will be considered. The velocity of the string is assumed to be constant, and the nonhomogeneous terms in the string equation are due to external force acting on the string. The Laplace transform method will be used to construct the solution of the problem. It will turn out that the method has considerable, computational advantages compared to the usually applied method of modal analysis based on eigenfunction expansions.
\end{abstract}

\footnotetext{
* Corresponding author. Tel.: +31-15-2783524; fax: +31-15-2787295.

Email addresses: W.T.vanHorssen@its.tudelft.nl (W.T. van Horssen ), svetlana@dutind4.twi.tudelft.nl (S.V. Ponomareva).
} 


\section{Introduction.}

The dynamics of axially moving materials have been studied by many researchers due to their technological importance. Transversal vibrations of belt systems have been investigated for many years. A lot of literature is devoted to this problem (see the reference lists in [1]- [5]). The main goal of this paper is to investigate a linear, nonhomogeneous equation for a moving string which is a starting point to study later weakly perturbed or weakly nonlinear problems for moving string equations. First the the displacement of the string in vertical direction will be determined by using the Laplace transform method. In the second part of this paper the solution will be constructed by using the method of eigenfunction expansions. This last method was developed for this problems by Meirovitch in [6,7] and by Wickert and Mote in [1]. Both methods will be compared. The conditions under which bounded solutions exist will be derived, and it will be shown for what kind of external forces internal resonances in the system will occur.

The following linear equation of motion for the string (moving in one direction with a constant velocity $V_{0}$ ) will be considered in this paper:

$$
u_{t t}+2 V_{0} u_{x t}+\left(V_{0}^{2}-c^{2}\right) u_{x x}=g(x, t), 0<x<l, t>0
$$


where

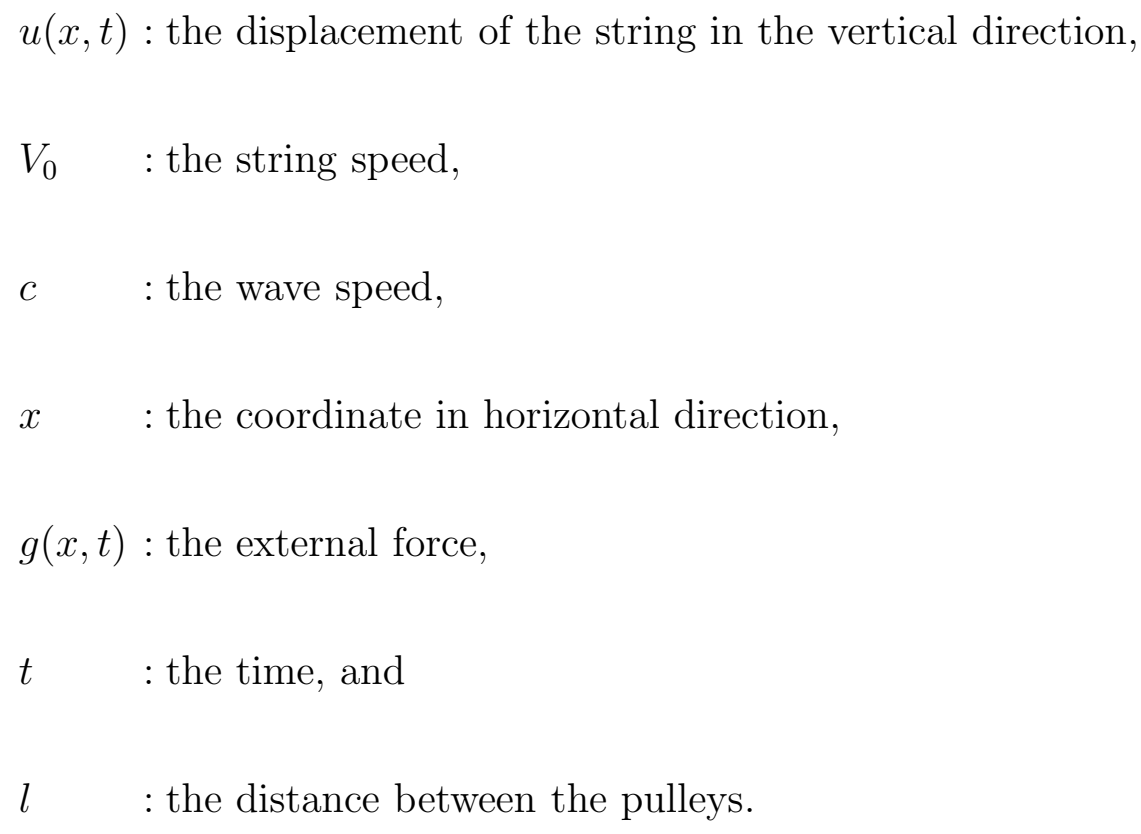

In this paper the case $V_{0}<c$ is considered. At the pulleys it is assumed that there is no displacement of the string in vertical direction. Equation (1) can also be found in [2], but now it is assumed that $V_{0}$ is not necessarily small. The boundary and initial conditions for $u(x, t)$ are given by:

$$
\begin{gathered}
u(0, t)=u(l, t)=0, t \geqslant 0, \\
u(x, 0)=f(x), \text { and } u_{t}(x, 0)=r(x), 0<x<l,
\end{gathered}
$$

where $f(x)$ and $r(x)$ represent the initial displacement and the initial velocity of the string, respectively. It is assumed that the functions $f(x)$ and $r(x)$ are sufficiently smooth such that a two times continuously differentiable solution for the initial-boundary value problem (1) - (2) exists. 


\section{Application of the Laplace transform method.}

The initial-boundary value problem (1) - (2) for $u(x, t)$ can readily be solved by applying the Laplace transform method (with respect to time $t$ ) to (1) (2), yielding:

$$
\begin{gathered}
s^{2} U(x, s)-s u(x, 0)-u_{t}(x, 0)+2 V_{0}\left(s U_{x}(x, s)-u_{x}(x, 0)\right)+ \\
U_{x x}(x, s)\left(V_{0}^{2}-c^{2}\right)=G_{1}(x, s), \\
U(0, s)=U(l, s)=0,
\end{gathered}
$$

where $U(x, s)$ and $G_{1}(x, s)$ are the Laplace transforms of $u(x, t)$ and $g(x, t)$ respectively.

By dividing (3) by $\left(V_{0}^{2}-c^{2}\right)$ and by rearranging terms in (3) it follows that:

$$
U_{x x}+\frac{2 V_{0} s}{V_{0}^{2}-c^{2}} U_{x}+\frac{s^{2}}{V_{0}^{2}-c^{2}} U=G(x, s),
$$

where

$$
G(x, s)=\frac{G_{1}(x, s)+s f(x)+r(x)+2 V_{0} f_{x}(x)}{V_{0}^{2}-c^{2}} .
$$

The general solution of the homogeneous equation (that is, (5) with $G \equiv 0$ ) is given by

$$
U(x, s)=C_{1}(s) \exp \left(\frac{-s x}{V_{0}+c}\right)+C_{2}(s) \exp \left(\frac{-s x}{V_{0}-c}\right)
$$

where $C_{1}(s)$ and $C_{2}(s)$ are still arbitrary functions. The method of variation of parameters can be used to find the particular solution of the nonhomogeneous equation (5). To apply this method the solution is written in the form

$$
U(x, s)=C_{1}(x, s) \exp \left(\frac{-s x}{V_{0}+c}\right)+C_{2}(x, s) \exp \left(\frac{-s x}{V_{0}-c}\right) .
$$


The functions $C_{1}(x, s)$ and $C_{2}(x, s)$ then follow from

$$
\left\{\begin{array}{l}
C_{1}^{\prime}(x, s) \exp \left(\frac{-s x}{V_{0}+c}\right)+C_{2}^{\prime}(x, s) \exp \left(\frac{-s x}{V_{0}-c}\right)=0, \\
C_{1}^{\prime}(x, s)\left(\frac{-s}{V_{0}+c}\right) \exp \left(\frac{-s x}{V_{0}+c}\right)+C_{2}^{\prime}(x, s)\left(\frac{-s}{V_{0}-c}\right) \exp \left(\frac{-s x}{V_{0}-c}\right)=G(x, s),
\end{array}\right.
$$

or equivalently from

$$
\left\{\begin{array}{l}
C_{1}^{\prime}(x, s)=G(x, s) \frac{\left(V_{0}^{2}-c^{2}\right)}{2 s c} \exp \left(\frac{s x}{V_{0}+c}\right) \\
C_{2}^{\prime}(x, s)=-G(x, s) \frac{\left(V_{0}^{2}-c^{2}\right)}{2 s c} \exp \left(\frac{s x}{V_{0}-c}\right)
\end{array}\right.
$$

where the prime' denotes differentiation with respect to $x$. By integrating (8) with respect to $x$ it follows that

$$
\left\{\begin{array}{l}
C_{1}(x, s)=\frac{\left(V_{0}^{2}-c^{2}\right)}{2 s c} \int_{0}^{x} G\left(x^{*}, s\right) \exp \left(\frac{x^{*} s}{V_{0}^{2}+c^{2}}\right) d x^{*}+K_{1}(s) \\
C_{2}(x, s)=-\frac{\left(V_{0}^{2}-c^{2}\right)}{2 s c} \int_{0}^{x} G\left(x^{*}, s\right) \exp \left(\frac{x^{*} s}{V_{0}^{2}-c^{2}}\right) d x^{*}+K_{2}(s)
\end{array}\right.
$$

where $K_{1}(s)$ and $K_{2}(s)$ are still arbitrary functions. The solution of the nonhomogeneous equation (3) or (5) is given by

$$
\begin{aligned}
U(x, s) & =K_{1}(s) \exp \left(\frac{-s x}{V_{0}-c}\right)+K_{2}(s) \exp \left(\frac{-s x}{V_{0}+c}\right)+ \\
& \frac{\left(V_{0}^{2}-c^{2}\right)}{2 s c} \int_{0}^{x} G\left(x^{*}, s\right)\left(\exp \left(\frac{-s\left(x-x^{*}\right)}{V_{0}+c}\right)-\exp \left(\frac{-s\left(x-x^{*}\right)}{V_{0}-c}\right)\right) d x^{*},
\end{aligned}
$$

where $K_{1}(s)$ and $K_{2}(s)$ can be determined from the boundary conditions (4). 
So, finally the following expression for $U(x, s)$ is found:

$$
\begin{aligned}
& U(x, s)= \\
& \frac{\left(V_{0}^{2}-c^{2}\right)}{2 s c} \frac{\int_{0}^{l} G\left(x^{*}, s\right)\left(\exp \left(\frac{-s\left(l-x^{*}\right)}{V_{0}+c}\right)-\exp \left(\frac{-s\left(l-x^{*}\right)}{V_{0}-c}\right)\right) d x^{*}}{\exp \left(\frac{-s l}{V_{0}+c}\right)-\exp \left(\frac{-s l}{V_{0}-c}\right)} \times \\
& \left(-\exp \left(\frac{-s x}{V_{0}+c}\right)+\exp \left(\frac{-s x}{V_{0}-c}\right)\right)+ \\
& \frac{\left(V_{0}^{2}-c^{2}\right)}{2 s c} \int_{0}^{x} G\left(x^{*}, s\right)\left(\exp \left(\frac{-s\left(x-x^{*}\right)}{V_{0}+c}\right)-\exp \left(\frac{-s\left(x-x^{*}\right)}{V_{0}-c}\right)\right) d x^{*} .
\end{aligned}
$$

The inverse Laplace transform of $U(x, s)$ is given by

$$
u(x, t)=\frac{1}{2 \pi i} \int_{\nu-i \infty}^{\nu+i \infty} U(x, s) e^{s t} d s=\sum_{n} \operatorname{Res}\left(s_{n}, x, t\right), \text { for some } \nu>0,
$$

and where Res stands for the residue at $s=s_{n}$. To evaluate the inverse Laplace transform $(11)$ the poles of $U(x, s)$ and the order of these poles have to be determined in the complex s-plane. As long as $g(x, t)$ is not specified the poles due to $G_{1}(x, s)$ will be unknown. For that reason three cases will be considered: (i) $g(x, t)=0$, (ii) $g(x, t)=\varphi(x) \sin (\omega t)$ with $\omega=\frac{\pi n^{*}}{l c}\left(V_{0}^{2}-c^{2}\right)$ for some fixed $n^{*} \in \mathbb{Z}$, and (iii) $g(x, t)=\varphi(x) \sin (\omega t)$ with $\omega$ is not in the neighborhood of $\frac{\pi n}{l c}\left(V_{0}^{2}-c^{2}\right)$ for all $n \in \mathbb{Z}$.

2.1. Case (i): $g(x, t)=0$.

In this case the poles of $U(x, s)$ follow from (see $(10))$

$$
s\left(\exp \left(\frac{-s l}{V_{0}+c}\right)-\exp \left(\frac{-s l}{V_{0}-c}\right)\right)=0 .
$$

Now it should be observed that $s=0$ is not a pole of $U(x, s)$ since $\lim _{s \rightarrow 0} U(x, s)$ exists. All other poles of $U(x, s)$ now follow from

$$
\exp \left(\frac{-s l}{V_{0}+c}\right)-\exp \left(\frac{-s l}{V_{0}-c}\right)=0
$$


and are given by

$$
s_{n}=\frac{\pi n}{l c}\left(V_{0}^{2}-c^{2}\right) i,
$$

with $n \in \mathbb{Z} \backslash\{0\}$. It should be observed that these poles are all simple. The solution of the initial-boundary value problem (1)-(2) with $g(x, t)=0$ now easily follows from (11), yielding

$$
\begin{aligned}
& u(x, t)= \\
& \sum_{n=1}^{\infty}\left\{a _ { n } \left(\cos \left(\frac{\pi n\left(V_{0}^{2}-c^{2}\right) t}{l c}\right)\left(\cos \left(\frac{\pi n\left(V_{0}+c\right) x}{l c}\right)-\cos \left(\frac{\pi n\left(V_{0}-c\right) x}{l c}\right)\right)+\right.\right. \\
& \left.\sin \left(\frac{\pi n\left(V_{0}^{2}-c^{2}\right) t}{l c}\right)\left(\sin \left(\frac{\pi n\left(V_{0}+c\right) x}{l c}\right)-\sin \left(\frac{\pi n\left(V_{0}-c\right) x}{l c}\right)\right)\right)+ \\
& b_{n}\left(\cos \left(\frac{\pi n\left(V_{0}^{2}-c^{2}\right) t}{l c}\right)\left(\sin \left(\frac{\pi n\left(V_{0}+c\right) x}{l c}\right)-\sin \left(\frac{\pi n\left(V_{0}-c\right) x}{l c}\right)\right)-\right. \\
& \left.\left.\sin \left(\frac{\pi n\left(V_{0}^{2}-c^{2}\right) t}{l c}\right)\left(\cos \left(\frac{\pi n\left(V_{0}+c\right) x}{l c}\right)-\cos \left(\frac{\pi n\left(V_{0}-c\right) x}{l c}\right)\right)\right)\right\}
\end{aligned}
$$

where

$$
\begin{aligned}
& a_{n}= \\
& \frac{\left(V_{0}^{2}-c^{2}\right)}{c}\left(\frac{1}{2 l c} \int_{0}^{l} f\left(x^{*}\right)\left(\cos \left(\frac{\left(V_{0}-c\right) \pi n x^{*}}{l c}\right)-\cos \left(\frac{\left(V_{0}+c\right) \pi n x^{*}}{l c}\right)\right) d x^{*}+\right. \\
& \left.\frac{1}{2} \int_{0}^{l} \frac{r\left(x^{*}\right)+2 V_{0} f_{x}^{\prime}\left(x^{*}\right)}{\pi n\left(V_{0}^{2}-c^{2}\right)}\left(\sin \left(\frac{\left(V_{0}-c\right) \pi n x^{*}}{l c}\right)-\sin \left(\frac{\left(V_{0}+c\right) \pi n x^{*}}{l c}\right)\right) d x^{*}\right) \\
& b_{n}= \\
& \frac{\left(V_{0}^{2}-c^{2}\right)}{c}\left(\frac{1}{2 l c} \int_{0}^{l} f\left(x^{*}\right)\left(\sin \left(\frac{\left(V_{0}-c\right) \pi n x^{*}}{l c}\right)-\sin \left(\frac{\left(V_{0}+c\right) \pi n x^{*}}{l c}\right)\right) d x^{*}-\right. \\
& \left.\frac{1}{2} \int_{0}^{l} \frac{r\left(x^{*}\right)+2 V_{0} f_{x}^{\prime}\left(x^{*}\right)}{\pi n\left(V_{0}^{2}-c^{2}\right)}\left(\cos \left(\frac{\left(V_{0}-c\right) \pi n x^{*}}{l c}\right)-\cos \left(\frac{\left(V_{0}+c\right) \pi n x^{*}}{l c}\right)\right) d x^{*}\right) .
\end{aligned}
$$

\subsection{Case (ii): $g(x, t)=\varphi(x) \sin (\omega t)$, the resonant case.}

In this case it is assumed that $\omega$ is equal to an natural frequency of the axially moving string, that is, $\omega=\frac{\pi n^{*}}{l c}\left(V_{0}^{2}-c^{2}\right)$ for some fixed $n^{*} \in \mathbb{Z}$. The Laplace 
transform of $g(x, t)$ in this case is

$$
G_{1}(x, s)=\frac{\omega}{s^{2}+\omega^{2}} \varphi(x)
$$

In $(10) G(x, s)$ is now given by $G\left(x^{*}, s\right)=\frac{\omega \varphi\left(x^{*}\right)}{\left(s^{2}+\omega^{2}\right)\left(V_{0}^{2}-c^{2}\right)}+h\left(x^{*}, s\right)$, where $h\left(x^{*}, s\right)=\frac{s f\left(x^{*}\right)+r\left(x^{*}\right)+2 V_{0} f_{x^{*}}\left(x^{*}\right)}{V_{0}^{2}-c^{2}}$. And so, $U(x, s)$ can be written as $U(x, s)=$ $A(x, s)+B(x, s)+D(x, s)+E(x, s)$, where

$$
\begin{aligned}
& A(x, s)= \\
& \frac{\left(V_{0}^{2}-c^{2}\right)}{2 s c} \frac{\omega \int_{0}^{l} \varphi\left(x^{*}\right)\left(\exp \left(\frac{-s\left(l-x^{*}\right)}{V_{0}+c}\right)-\exp \left(\frac{-s\left(l-x^{*}\right)}{V_{0}-c}\right)\right) d x^{*}}{\left(s^{2}+\omega^{2}\right)\left(V_{0}^{2}-c^{2}\right)\left(\exp \left(\frac{-s l}{V_{0}+c}\right)-\exp \left(\frac{-s l}{V_{0}-c}\right)\right)} \times \\
& \left(-\exp \left(\frac{-s x}{V_{0}+c}\right)+\exp \left(\frac{-s x}{V_{0}-c}\right)\right) \text {, } \\
& B(x, s)= \\
& \frac{\left(V_{0}^{2}-c^{2}\right)}{2 s c} \frac{\int_{0}^{l} h\left(x^{*}, s\right)\left(\exp \left(\frac{-s\left(l-x^{*}\right)}{V_{0}+c}\right)-\exp \left(\frac{-s\left(l-x^{*}\right)}{V_{0}-c}\right)\right) d x^{*}}{\left(\exp \left(\frac{-s l}{V_{0}+c}\right)-\exp \left(\frac{-s l}{V_{0}-c}\right)\right)} \times \\
& \left(-\exp \left(\frac{-s x}{V_{0}+c}\right)+\exp \left(\frac{-s x}{V_{0}-c}\right)\right) \text {, } \\
& D(x, s)= \\
& \frac{\omega}{2 s c\left(s^{2}+\omega^{2}\right)} \int_{0}^{x} \varphi\left(x^{*}\right)\left(\exp \left(\frac{-s\left(x-x^{*}\right)}{V_{0}+c}\right)-\exp \left(\frac{-s\left(x-x^{*}\right)}{V_{0}-c}\right)\right) d x^{*}, \\
& E(x, s)= \\
& \frac{\left(V_{0}^{2}-c^{2}\right)}{2 s c} \int_{0}^{x} h\left(x^{*}, s\right)\left(\exp \left(\frac{-s\left(x-x^{*}\right)}{V_{0}+c}\right)-\exp \left(\frac{-s\left(x-x^{*}\right)}{V_{0}-c}\right)\right) d x^{*} .
\end{aligned}
$$

The inverse Laplace transform of $U(x, s)$ is given by

$$
L^{i n v}(U(x, s))=L^{i n v}(A(x, s))+L^{i n v}(B(x, s))+L^{i n v}(D(x, s))+L^{i n v}(E(x, s)) .
$$

In $(18) L^{i n v}(B(x, s))$ and $L^{i n v}(E(x, s))$ only depend on the initial values $f(x)$ and $r(x)$ and already have been determined in case (i) with $g(x, t)=0$. So, only 
$L^{i n v}(A(x, s))$ and $L^{i n v}(D(x, s))$ have to be calculated. It should be observed that these inverse Laplace transforms only depend on $g(x, t)$. Furthermore, it should be observed that $A(x, s)$ is the product of $\frac{\omega}{s^{2}+\omega^{2}}$ and another term (following from (14)), and so the inverse Laplace transform of $A(x, s)$ can be determined by using the convolution integral, that is,

$$
L^{i n v}(A(x, s))=\int_{0}^{t} \sin (\omega(t-\tau)) \sum_{n} \operatorname{Res}\left(s_{n}, x, \tau\right) d \tau
$$

where $s_{n}$ is given by (13). Finally, if $\omega=\frac{\pi n^{*}}{l c}\left(V_{0}^{2}-c^{2}\right)$ for a fixed $n^{*} \in \mathbb{Z}$ it follows that $L^{i n v}(A(x, s))$ is

$$
\begin{aligned}
& L^{i n v}(A(x, s))=\frac{F_{1 n^{*}}(x)}{2} t \sin \left(\omega_{n^{*}} t\right)+\frac{F_{2 n^{*}}(x)}{2}\left(\frac{\sin \left(\omega_{n^{*}} t\right)}{\omega_{n^{*}}}-t \cos \left(\omega_{n^{*}} t\right)\right)+ \\
& \sum_{n=1, n \neq\left|n^{*}\right|}^{\infty}\left\{\frac { F _ { 1 n } ( x ) } { 2 } \left(\frac{1}{\omega_{n^{*}}-\omega_{n}}\left(\cos \left(\omega_{n} t\right)-\cos \left(\omega_{n^{*}} t\right)\right)+\right.\right. \\
& \left.\frac{1}{\omega_{n^{*}}+\omega_{n}}\left(\cos \left(\omega_{n} t\right)-\cos \left(\omega_{n^{*}} t\right)\right)\right)+ \\
& \left.\frac{F_{2 n}(x)}{2}\left(\frac{1}{\omega_{n^{*}}+\omega_{n}}\left(\sin \left(\omega_{n} t\right)+\sin \left(\omega_{n^{*}} t\right)\right)+\frac{1}{\omega_{n^{*}}-\omega_{n}}\left(\sin \left(\omega_{n} t\right)-\sin \left(\omega_{n^{*}} t\right)\right)\right)\right),
\end{aligned}
$$

where

$$
\begin{gathered}
F_{1 n}(x)=w_{n}\left(\cos \left(\frac{\pi n\left(V_{0}+c\right) x}{l c}\right)-\cos \left(\frac{\pi n\left(V_{0}-c\right) x}{l c}\right)\right)+ \\
p_{n}\left(\sin \left(\frac{\pi n\left(V_{0}+c\right) x}{l c}\right)-\sin \left(\frac{\pi n\left(V_{0}-c\right) x}{l c}\right)\right), \\
F_{2 n}(x)=w_{n}\left(\sin \left(\frac{\pi n\left(V_{0}+c\right) x}{l c}\right)-\sin \left(\frac{\pi n\left(V_{0}-c\right) x}{l c}\right)\right)- \\
p_{n}\left(\cos \left(\frac{\pi n\left(V_{0}+c\right) x}{l c}\right)-\cos \left(\frac{\pi n\left(V_{0}-c\right) x}{l c}\right)\right), \\
w_{n}=\frac{1}{2 c} \int_{0}^{l} \frac{\varphi\left(x^{*}\right)}{\pi n}\left(\sin \left(\frac{\left(V_{0}-c\right) \pi n x^{*}}{l c}\right)-\sin \left(\frac{\left(V_{0}+c\right) \pi n x^{*}}{l c}\right)\right) d x^{*}, \\
p_{n}=\frac{1}{2 c} \int_{0}^{l} \frac{\varphi\left(x^{*}\right)}{\pi n}\left(\cos \left(\frac{\left(V_{0}-c\right) \pi n x^{*}}{l c}\right)-\cos \left(\frac{\left(V_{0}+c\right) \pi n x^{*}}{l c}\right)\right) d x^{*} .
\end{gathered}
$$

It can be seen from (14) that if $n=n^{*}$ then there are two poles of order two (one in $s=i \omega_{n^{*}}$ and one in $\left.s=-i \omega_{n^{*}}\right)$. To calculate $L^{i n v}(D(x, s)$ ) it should be 
observed that $s=0$ is not a pole as $\lim _{s \rightarrow 0} D(x, s)$ exists. So the inverse Laplace transform for $D(x, s)$ is

$$
\begin{aligned}
& L^{i n v}(D(x, s))= \\
& \frac{\left(V_{0}^{2}-c^{2}\right)}{c \omega}\left(\frac{1}{2} \cos (\omega t) \int_{0}^{x} \varphi\left(x^{*}\right)\left(\cos \left(\frac{\omega\left(x-x^{*}\right)}{V_{0}+c}\right)-\cos \left(\frac{\omega\left(x-x^{*}\right)}{V_{0}+c}\right)\right) d x^{*}+\right. \\
& \left.\frac{1}{2} \sin (\omega t) \int_{0}^{x} \varphi\left(x^{*}\right)\left(\sin \left(\frac{\omega\left(x-x^{*}\right)}{V_{0}+c}\right)-\sin \left(\frac{\omega\left(x-x^{*}\right)}{V_{0}+c}\right)\right) d x^{*}\right) .
\end{aligned}
$$

It can be seen that only $L^{i n v}(A(x, s))$ contributes to unbounded terms in the solution.

2.3. Case (iii): $g(x, t)=\varphi(x) \sin (\omega t)$, the non-resonant case.

Let $\omega_{n}=\frac{\pi n}{l c}\left(V_{0}^{2}-c^{2}\right), n \in \mathbb{Z}$ be the natural frequencies of an axially moving string and let $\omega$ be not in a neighborhood of any of these frequencies $\omega_{n}$.

For $L^{i n v}(A(x, s))$ it then follows that

$$
\begin{aligned}
& L^{\text {inv }}(A(x, s))= \\
& \sum_{n=1}^{\infty}\left(\frac{F_{1 n}(x)}{2}\left(\frac{1}{\omega-\omega_{n}}\left(\cos \left(\omega_{n} t\right)-\cos (\omega t)\right)+\frac{1}{\omega+\omega_{n}}\left(\cos \left(\omega_{n} t\right)-\cos (\omega t)\right)\right)+\right. \\
& \left.\frac{F_{2 n}(x)}{2}\left(\frac{1}{\omega+\omega_{n}}\left(\sin \left(\omega_{n} t\right)+\sin (\omega t)\right)+\frac{1}{\omega-\omega_{n}}\left(\sin \left(\omega_{n} t\right)-\sin (\omega t)\right)\right)\right),
\end{aligned}
$$

where $F_{1 n}(x)$ and $F_{1 n}(x)$ are given by (21) and (22), respectively. $L^{i n v}(D(x, s))$ is again given by (25). In this case there are no unbounded terms in the solution $u(x, t)$. Obviously, unbounded solutions will occur when $g(x, t)$ contains terms $\varphi_{1}(x) \sin (\omega t)$ and/or terms $\varphi_{2}(x) \cos (\omega t)$ for which $\omega$ is equal to an natural frequency $\omega_{n}$. 


\section{An eigenfunction approach.}

Following Wickert and Mote [1] equation (1) can be rewritten in the following dimensionless form:

$$
u_{t t}+2 \nu u_{x t}-\left(1-\nu^{2}\right) u_{x x}=g(x, t), 0<x<1, t>0
$$

where $\nu=\frac{V_{0}}{c}$. Introducing the operators $M=1, G=2 \nu \frac{\partial}{\partial x}$ and $K=-(1-$ $\left.\nu^{2}\right) \frac{\partial^{2}}{\partial x^{2}}$ in $(27)$ it follows that

$$
M u_{t t}+G u_{t}+K u=g(x, t) .
$$

Now the following vectors and matrices are defined

$$
\begin{gathered}
\mathbf{w}(x, t)=\left(\begin{array}{c}
u_{t}(x, t) \\
u(x, t)
\end{array}\right), \quad \mathbf{q}(x, t)=\left(\begin{array}{c}
g(x, t) \\
0
\end{array}\right), \\
\mathbf{A}=\left(\begin{array}{cc}
M & 0 \\
0 & K
\end{array}\right), \quad \text { and } \mathbf{B}=\left(\begin{array}{cc}
G & K \\
-K & 0
\end{array}\right) .
\end{gathered}
$$

Equation (28) then becomes

$$
\mathbf{A} \mathbf{w}_{t}+\mathbf{B w}=\mathbf{q}
$$

The solution of equation (31) has to satisfy the initial and the boundary conditions 


$$
\mathbf{w}(x, 0)=\mathbf{w}_{0}(x)=\left(\begin{array}{c}
r(x) \\
f(x)
\end{array}\right), \quad \text { and } \quad \mathbf{w}(x, t)=\mathbf{0} \quad \text { at } \quad x=0 \quad \text { and } \quad x=1 .
$$

The inner product of two vectors $\mathbf{w}_{1}$ and $\mathbf{w}_{2}$ is defined to be

$$
\left\langle\mathbf{w}_{1}, \mathbf{w}_{2}\right\rangle=\int_{0}^{1} \mathbf{w}_{1}^{T} \mathbf{w}_{2}^{*} d x
$$

where the * means the complex conjugate, and where the superscript $T$ means the transpose. With respect to this inner product the operators $\mathbf{A}$ and $\mathbf{B}$ have the following properties

$$
\left\langle\mathbf{A} \mathbf{w}_{1}, \mathbf{w}_{2}\right\rangle=\left\langle\mathbf{w}_{1}, \mathbf{A} \mathbf{w}_{2}\right\rangle,\left\langle\mathbf{B w}_{1}, \mathbf{w}_{2}\right\rangle=-\left\langle\mathbf{w}_{1}, \mathbf{B w}_{2}\right\rangle
$$

where both $\mathbf{w}_{1}(\mathrm{x}, \mathrm{t})$ and $\mathbf{w}_{2}(\mathrm{x}, \mathrm{t})$ satisfy the Dirichlet boundary conditions $(32)$ at $x=0$ and $x=1$. The general solution of the equation (31) will be found in an expansion of eigenfunctions. First the homogeneous equation (31) with $\mathbf{q}=0$ is solved by using the method of separation of variables, that is, it is assumed that (31) with $\mathbf{q}=0$ admits solution of the form $\phi(x) \exp (\lambda t)$, where $\phi(x)$ is a complex vector function and where $\lambda$ is a complex-valued number . This leads to the eigenvalue problem

$$
\lambda \mathbf{A} \phi+\mathbf{B} \phi=0
$$

where the eigenvalues $\lambda_{n}$ are equal to $i \omega_{n}$ as has been shown by Meirovitch in [6]. The complex-valued eigenfunctions $\phi_{n}$ have the structure $\phi_{n}(x)=$ 
$\phi_{n}^{R}(x)+i \phi_{n}^{I}(x)$, where

$$
\boldsymbol{\phi}_{n}^{R}(x)=\left(\begin{array}{c}
-\omega_{n} \psi_{n}^{I}(x) \\
\psi_{n}^{R}(x)
\end{array}\right) \text { and } \phi_{n}^{I}(x)=\left(\begin{array}{c}
\omega_{n} \psi_{n}^{R}(x) \\
\psi_{n}^{I}(x)
\end{array}\right) .
$$

In $(36) \psi_{n}(x)$ is the complex normalized scalar eigenfunction and $\psi_{n}(x)=$ $\psi_{n}^{R}(x)+i \psi_{n}^{I}(x)$. After solving the eigenvalue problem (35) and after normalization the following expressions can be found

$$
\begin{gathered}
\omega_{n}=n \pi\left(1-\nu^{2}\right), \\
\psi_{n}^{R}(x)=\frac{1}{n \pi} \sqrt{\frac{2}{1-\nu^{2}}} \sin (n \pi x) \cos (n \pi \nu x), \\
\psi_{n}^{I}(x)=\frac{1}{n \pi} \sqrt{\frac{2}{1-\nu^{2}}} \sin (n \pi x) \sin (n \pi \nu x) .
\end{gathered}
$$

The normalization requirements can be found in [1]. The $\phi_{n}(x)$ satisfy the orthonormality relations

$$
\begin{aligned}
& \left\langle\mathbf{A} \phi_{n}^{R}, \phi_{m}^{R}\right\rangle=\delta_{n m},\left\langle\mathbf{A} \phi_{n}^{I}, \phi_{m}^{I}\right\rangle=\delta_{n m},\left\langle\mathbf{A} \phi_{n}^{R}, \phi_{m}^{I}\right\rangle=0, \\
& \left\langle\mathbf{B} \phi_{n}^{R}, \phi_{m}^{R}\right\rangle=0, \quad\left\langle\mathbf{B} \phi_{n}^{I}, \phi_{m}^{I}\right\rangle=0, \quad\left\langle\mathbf{B} \phi_{n}^{R}, \phi_{m}^{I}\right\rangle=\omega_{n} \delta_{n m},
\end{aligned}
$$

where $\delta_{n m}$ is the Kronecker delta symbol, that is, $\delta_{n m}=1$ for $n=m$ and zero otherwise. It was shown by Meirovitch in [7] that the solution of the nonhomogeneous equation (31) can be found in the following form

$$
\mathbf{w}(x, t)=\sum_{n=1}^{\infty} \xi_{n}^{R}(t) \boldsymbol{\phi}_{n}^{R}(x)+\xi_{n}^{I}(t) \boldsymbol{\phi}_{n}^{I}(x),
$$

where $\xi_{n}^{I}(t)$ and $\xi_{n}^{R}(t)$ are the components of the so-called generalized coordi- 
nates. Substituting this expansion into equation (31) it follows that

$$
\mathbf{A} \frac{\partial}{\partial t} \sum_{n=1}^{\infty}\left[\xi_{n}^{R}(t) \boldsymbol{\phi}_{n}^{R}(x)+\xi_{n}^{I}(t) \boldsymbol{\phi}_{n}^{I}(x)\right]+\mathbf{B} \sum_{n=1}^{\infty}\left[\xi_{n}^{R}(t) \boldsymbol{\phi}_{n}^{R}(x)+\xi_{n}^{I}(t) \boldsymbol{\phi}_{n}^{I}(x)\right]=\mathbf{q}
$$

By multiplying (41) consecutively first with $\phi_{m}^{R}$ and then with $\phi_{m}^{I}$ and by integrating the so-obtained equations with respect to $x$ from $x=0$ to $x=$ 1 in the sense of the defined inner product (33) and with the help of the orthonormality relations (39) the following system is obtained

$$
\left\{\begin{array}{l}
\frac{d \xi_{m}^{R}}{d t}-\omega_{m} \xi_{m}^{I}=-\omega_{m} \int_{0}^{1} \psi_{m}^{I} g(x, t) d x \\
\frac{d \xi_{m}^{I}}{d t}+\omega_{m} \xi_{m}^{R}=\omega_{m} \int_{0}^{1} \psi_{m}^{R} g(x, t) d x
\end{array}\right.
$$

Solving this system with respect to the initial values $\xi_{m}^{R}(0)=\left\langle\mathbf{A w}_{0}, \boldsymbol{\phi}_{m}^{R}\right\rangle$ and $\xi_{m}^{I}(0)=\left\langle\mathbf{A w}_{0}, \phi_{m}^{I}\right\rangle$ it follows that

$$
\begin{gathered}
\xi_{m}^{R}(t)=\int_{0}^{t}\left(g_{m}^{R}(s) \cos \left(\omega_{m}(t-s)\right)+g_{m}^{I}(s) \sin \left(\omega_{m}(t-s)\right)\right) d s+ \\
\xi_{m}^{R}(0) \cos \left(\omega_{m} t\right)+\xi_{m}^{I}(0) \sin \left(\omega_{m} t\right), \\
\xi_{m}^{I}(t)=\int_{0}^{t}\left(g_{m}^{I}(s) \cos \left(\omega_{m}(t-s)\right)-g_{m}^{R}(s) \sin \left(\omega_{m}(t-s)\right)\right) d s+ \\
\xi_{m}^{I}(0) \cos \left(\omega_{m} t\right)-\xi_{m}^{R}(0) \sin \left(\omega_{m} t\right),
\end{gathered}
$$

where

$$
\begin{aligned}
& g_{m}^{R}(t)=-\sqrt{2\left(1-\nu^{2}\right)} \int_{0}^{1} g(x, t) \sin (m \pi x) \sin (m \pi \nu x) d x \text { and } \\
& g_{m}^{I}(t)=\sqrt{2\left(1-\nu^{2}\right)} \int_{0}^{1} g(x, t) \sin (m \pi x) \cos (m \pi \nu x) d x
\end{aligned}
$$


are defined to be the generalized forces. And $\xi_{m}^{R}(0), \xi_{m}^{I}(0)$ are given by

$$
\begin{aligned}
& \xi_{m}^{R}(0)= \\
& m \pi \sqrt{2\left(1-\nu^{2}\right)} \int_{0}^{1} f(x)\left(\left(1+\nu^{2}\right) \sin (m \pi x) \cos (m \pi \nu x)+\right. \\
& 2 \nu \cos (m \pi x) \sin (m \pi \nu x)) d x- \\
& \sqrt{2\left(1-\nu^{2}\right)} \int_{0}^{1} r(x) \sin (m \pi x) \sin (m \pi \nu x) d x \\
& \xi_{m}^{I}(0)= \\
& m \pi \sqrt{2\left(1-\nu^{2}\right)} \int_{0}^{1} f(x)\left(\left(1+\nu^{2}\right) \sin (m \pi x) \sin (m \pi \nu x)-\right. \\
& 2 \nu \cos (m \pi x) \cos (m \pi \nu x)) d x+ \\
& \sqrt{2\left(1-\nu^{2}\right)} \int_{0}^{1} r(x) \sin (m \pi x) \cos (m \pi \nu x) d x
\end{aligned}
$$

Using the definition (29) the expansion for $u(x, t)$ can be written in the form

$$
u(x, t)=\sum_{m=1}^{\infty} \xi_{m}^{R}(t) \psi_{m}^{R}(x)+\xi_{m}^{I}(t) \psi_{m}^{I}(x)
$$

or equivalently

$$
\begin{aligned}
& u(x, t)= \\
& \sum_{m=1}^{\infty} \frac{1}{m \pi} \sqrt{\frac{2}{1-\nu^{2}}}\left(\xi_{m}^{R}(t) \sin (m \pi x) \cos (m \pi \nu x)+\xi_{m}^{I}(t) \sin (m \pi x) \sin (m \pi \nu x)\right) .
\end{aligned}
$$


And, finally, the solution of the problem has the form:

$$
\begin{aligned}
& u(x, t)=\sum_{m=1}^{\infty} \frac{1}{m \pi} \sqrt{\frac{2}{1-\nu^{2}}} \times \\
& \left\{\left[\int _ { 0 } ^ { t } \left\{\left(-\sqrt{2\left(1-\nu^{2}\right)} \int_{0}^{1} g(x, s) \sin (m \pi x) \sin (m \pi \nu x) d x\right) \cos \left(\omega_{m}(t-s)\right)+\right.\right.\right. \\
& \left.\left(\sqrt{2\left(1-\nu^{2}\right)} \int_{0}^{1} g(x, s) \sin (m \pi x) \cos (m \pi \nu x) d x\right) \sin \left(\omega_{m}(t-s)\right)\right\} d s+ \\
& \left.\xi_{m}^{R}(0) \cos \left(\omega_{m} t\right)+\xi_{m}^{I}(0) \sin \left(\omega_{m} t\right)\right] \sin (m \pi x) \cos (m \pi \nu x)+ \\
& {\left[\int _ { 0 } ^ { t } \left\{\left(-\sqrt{2\left(1-\nu^{2}\right)} \int_{0}^{1} g(x, s) \sin (m \pi x) \cos (m \pi \nu x) d x\right) \cos \left(\omega_{m}(t-s)\right)+\right.\right.} \\
& \left.\left(\sqrt{2\left(1-\nu^{2}\right)} \int_{0}^{1} g(x, s) \sin (m \pi x) \sin (m \pi \nu x) d x\right) \sin \left(\omega_{m}(t-s)\right)\right\} d s+ \\
& \left.\left.\xi_{m}^{I}(0) \cos \left(\omega_{m} t\right)-\xi_{m}^{R}(0) \sin \left(\omega_{m} t\right)\right] \sin (m \pi x) \sin (m \pi \nu x)\right\} .
\end{aligned}
$$

From the structure of the solution as given by (48) it can be seen that if $g(x, t)=\varphi(x) \sin (\omega t)$ with $\omega=\omega_{m^{*}}$ for a fixed $m^{*} \in \mathbb{Z}$ resonance will occur (that is, unbounded solutions will occur). To show this two cases will be studied: case (a) $\omega=m^{*} \pi\left(1-\nu^{2}\right)$ and case (b) $\omega$ is not in a neighborhood of $\omega=m \pi\left(1-\nu^{2}\right)$ for all $m \in \mathbb{Z}$.

3.1. Case (a): $\omega=m^{*} \pi\left(1-\nu^{2}\right)$ for a fixed $m^{*} \in \mathbb{Z}$, the resonant case. 
The solution of the problem in this case is given by

$$
\begin{aligned}
& u(x, t)=\frac{1}{m^{*} \pi}\left\{t \operatorname { s i n } ( \omega _ { m ^ { * } } t ) \left(-\int_{0}^{1} H_{1 m^{*}}(x) d x \sin \left(m^{*} \pi x\right) \cos \left(m^{*} \pi \nu x\right)+\right.\right. \\
& \left.\int_{0}^{1} H_{2 m^{*}}(x) d x \sin \left(m^{*} \pi x\right) \sin \left(m^{*} \pi \nu x\right)\right)+\left(\frac{\sin \left(\omega^{m^{*}} t\right)}{\omega_{m^{*}}}-t \cos \left(\omega_{m^{*}} t\right)\right) \times \\
& \left(\int_{0}^{1} H_{2 m^{*}}(x) d x \sin \left(m^{*} \pi x\right) \cos \left(m^{*} \pi \nu x\right)+\right. \\
& \left.\left.\int_{0}^{1} H_{1 m^{*}}(x) d x \sin \left(m^{*} \pi x\right) \sin \left(m^{*} \pi \nu x\right)\right)\right\}+ \\
& \sum_{m=1, m \neq\left|m^{*}\right|}^{\infty}\left\{\frac { 2 } { m \pi } \left[\int_{0}^{1} H_{1 m^{*}}(x) d x\left(\frac{\omega\left(\cos (\omega t)-\cos \left(\omega_{m} t\right)\right)}{\omega^{2}-\omega_{m}^{2}}\right)+\right.\right. \\
& \int_{0}^{1} H_{2 m^{*}}(x) d x\left(\frac{\omega \sin \left(\omega_{m} t\right)-\omega_{m} \sin (\omega t)}{\omega^{2}-\omega_{m}^{2}}\right)+\xi_{m}^{R}(0) \cos \left(\omega_{m} t\right)+ \\
& \left.\xi_{m}^{I}(0) \sin \left(\omega_{m} t\right)\right] \sin (m \pi x) \sin (m \pi \nu x)+ \\
& {\left[\int_{0}^{1} H_{2 m^{*}}(x) d x\left(\frac{\omega\left(\cos \left(\omega_{m} t\right)-\cos (\omega t)\right)}{\omega^{2}-\omega_{m}^{2}}\right)+\right.} \\
& \int_{0}^{1} H_{1 m^{*}}(x) d x\left(\frac{\omega \sin \left(\omega_{m} t\right)-\omega_{m} \sin (\omega t)}{\omega^{2}-\omega_{m}^{2}}\right)+ \\
& \left.\left.\xi_{m}^{I}(0) \cos \left(\omega_{m} t\right)-\xi_{m}^{R}(0) \sin \left(\omega_{m} t\right)\right] \sin (m \pi x) \sin (m \pi \nu x)\right\}
\end{aligned}
$$

where

$$
\begin{aligned}
& H_{1 m}(x)=\varphi(x) \sin (m \pi x) \sin (m \pi \nu x), \\
& H_{2 m}(x)=\varphi(x) \sin (m \pi x) \cos (m \pi \nu x),
\end{aligned}
$$

and $\xi_{m}^{R}(0), \xi_{m}^{I}(0)$ are given by $(45)$.

3.2. Case $(b): g(x, t)=\varphi(x) \sin (\omega t)$, the non-resonant case.

It can easily be seen from (48) that if $\omega$ is not in a neighborhood of a natural frequency $\omega_{m}$ for all $m \in \mathbb{Z}$ then there are no unbounded terms in time in the solution. Finally it should be observed (by using trigonometric relations) that the solution as obtained in section 2 of this paper and in section 3 are identical. 


\section{Conclusions and remarks.}

In this paper an initial-boundary value problem for a linear, nonhomogeneous axially moving string equation has been studied. The velocity of the string is assumed to be constant, and the nonhomogeneous terms in the string equation are due to external forces acting on the string. To solve the initial-boundary value problem two methods are presented. One of these methods is the method of modal analysis based on eigenfunction expansions. This method has been introduced in [6,7] and in [1], and is used nowadays frequently for these types of problems (see for instance $[3,4]$ ). To apply this method an operator notation has to be introduced, an inner product has to be defined, an eigenvalue problem has to be solved, and orthonormality relations have to be determined. Altogether this method is rather complicated to apply to these types of problems. For that reason in this paper it is proposed to apply the Laplace transform method to these types of problems. When this method is applied poles (and the order of the poles) have to be determined, residues have to be calculated, and Cauchy's theorem has to be used (that is, integrals have to be evaluated by using the theory complex variables). To construct the solution by using the Laplace transform method is rather straightforward and more easy than the use of the method of modal analysis based on eigenfunction expansions. Moreover, the Laplace transform method is nowadays well-described in elementary textbooks on partial differential equations (see for instance [8]). In forthcoming papers it will be shown that the Laplace transform method can efficiently and easily be applied to weakly perturbed or weakly nonlinear (axially moving) string equations. 


\section{References}

[1] G. A. Wickert and C. D. Mote, Jr., Classical Vibration Analysis of Axially Moving Continua, Journal of Applied Mechanics 57 (1990) 738-744.

[2] G. Suweken and W.T. van Horssen, On The Transversal Vibrations of A Conveyor Belt with A Low and Time-Varying Velocity, Part I: The String-Like Case, Journal of Sound and Vibration 264 (2003) 117-133.

[3] H. R. Öz and M. Pakdemirli, Stability Analysis of An Axially Accelerating String, Journal of Sound and Vibration 227(2) (1999) 239-257.

[4] M. Pakdemirli and A. G. Ulsoy, Stability Analysis of An Axially Accelerating String, Journal of Sound and Vibration 203(5) (1997) 815-832.

[5] Li-Qun Chen, Jean W. Zu, Jun Wu and Xiao-Dong Yang, Transverse Vibrations of An Axially Accelerating Viscoelastic String with Geometric Nonlinearity, Journal of Engineering Mathematics 48(2) (2004) 117-182.

[6] L. Meirovitch, A New Method of Solution of The Eigenvalue Problem for Gyroscopic Systems, AIAA Journal 12 (1974) 1337-1342.

[7] L. Meirovitch, A Modal Analysis for the Response of Linear Gyroscopic Systems, ASME Journal of Applied Mechanics 42 (2) (1975) 446-450.

[8] R. Haberman, Applied Partial Differential Equations (Pearson Prentice Hall, New Jersey, 2004) 


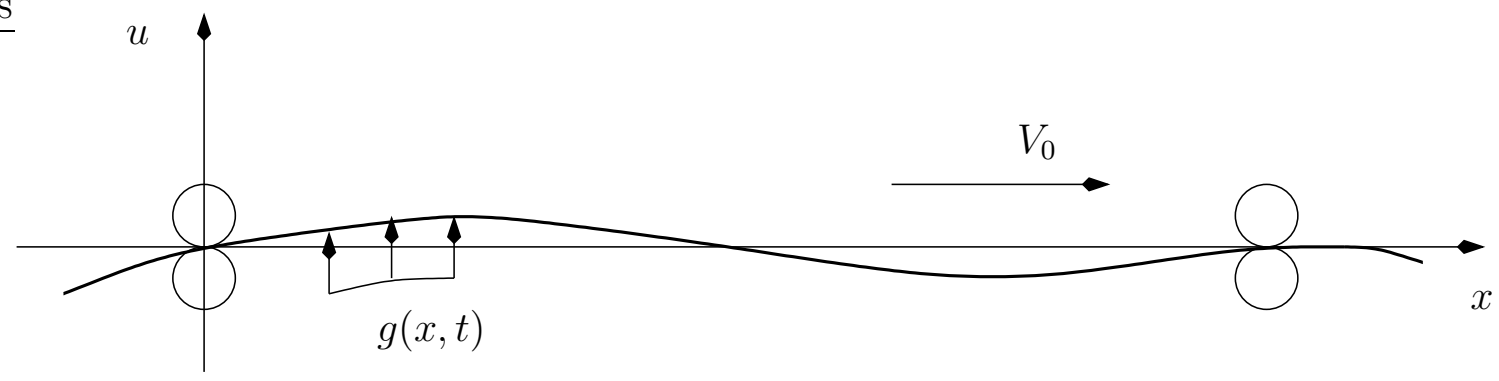

Fig. 1. An axially moving string 